\title{
SOBRE ALGUNS NÍVEIS DE CORRELACÃO BIOESTRATIGRÁFICA REGIONAL (FORAMINÍFEROS PLANCTÔNICÓS) NO CRETÁCEO BRASILEIRO
}

\author{
MARTA CLÁUDIA VIVIERS* e GERHARD BEURLEN*
}

\begin{abstract}
Six Cretaceous planktonic foraminiferal extinction levels allow good interbasinal correlation along the Brazilian continental margin. From base to top they are as follows: extinction of Favusella and Ticinella at the base and top of the Vraconian, respectively; extinction at Late Turonian of Globotruncana inornata and G. wilsoni in equatorial marginal basins and of Dicarinella hagni and D. imbricata in the Santos and Campos basins on eastern margin; the extinction of Dicarinella concavata at the Santonian-Campanian boundary; the extinction of Globotruncana fornicata at the Early-Late Maastrichtian. Three significant regional unconformities have been identified during Cretaceous marine sedimentation. The lack of Late Campanian and Latest Maastrichtian-Earliest Paleocene sediments indicates a hiatus during these time intervals, caused, probably, by sea-level oscillations as suggested by erosional truncation of Santonian-lower Campanian and lower-upper Maastrichtian sediments. The third unconformity, probably controled by tectonic and sedimentary factors related to the post-rift stage 'of the opening of the South/Equatorial Atlantic ocean, is represented by lack of Cenomanian sediments.
\end{abstract}

INTRODUÇÃO Desde o estabelecimento do primeiro zoneamento bioestratigráfico do Cretáceo (foraminíferos planctônicos) para a margem continental brasileira (Noguti \& Dos Santos 1978), o qual representava um desenvolvimento do esquema proposto para a Bacia de Sergipe/Alagoas (Troelsen apud Schaller 1969), acumularam-se numerosos novos dados. Sua análise mostra que, muito embora o arcabouço bioestratigráfico geral dos grandes intervalos, quais sejam Albiano-Cenomaniano Inferior, Turoniano-Santoniano e Campaniano-Maastrichtiano, permaneça mais ou menos inalterado, uma série de subdivisões novas pode ser estabelecida para cada um deles, reflexo dos sistemas deposicionais peculiares a cada uma das bacias.

A introdução de métodos estratigráficos mais sofisticados no final da década de 70 (sismoestratigrafia, análise geoistórica) permitiu a utilização e interpretação mais objetiva, com um enfoque mais "geológico", dos dados paleontológicos e paleoecológicos existentes. Esta reanálise permitiu, pela primeira vez, uma visualização regional do desenvolvimento bioestratigráfico e paleoambiental do Cretáceo (Beurlen 1982), evidenciando, simultaneamente, uma série de problemas de correlação intra- e inter-regionais.

A partir daí, o Setor de Bioestratigrafia e Paleoecologia da Divisão de Exploração do Centro de Pesquisas e Desenvolvimento Leopoldo A. Miguez de Mello da Petróleo Brasileiro S.A. desenvolveu uma série de trabalhos visando a solução destes problemas, sendo os primeiros deles uma reanálise bioestratigráfica da Bacia do Ceará com palinomorfos (Regali 1980) e foraminíferos planctônicos (Viviers 1982). Seguiu-se o reestudo de outras áreas, acompanhando o programa de reavaliação de bacias levado a termo pelo Departamento de Exploração. Alguns dos resultados bioestratigráficos são apresentados nos trabalhos de Azevedo et al. (1987), Dias-Brito (1987), Koutsoukos (1987), Richter (1987), Viviers (1987) e Viviers \& Regali (1987).

Ao contrário dos trabalhos citados, este enfoca a correlação bioestratigráfica entre bacias com ênfase em data de foraminíferos planctônicos de validade mundial, bem como a análise de hiatos de extensão inter-regional. Os dados aqui discutidos provêm, em sua maioria, de poços perfurados nas bacias do Maranhão, Ceará e Potiguar, da margem equatorial e das de Campos e Santos na margem leste (Fig. 1).

\section{NIVEIS DE CORRELAÇÃO BIOESTRATIGRÁFICA IN-} TER-REGIONAL Seis data de foraminíferos planctônicos ocorrem no Cretáceo ao longo da margem continental brasileira. Permitem a datação bioestratigráfica das grandes seqüências sedimentares marinhas cretáceas (Ponte et al. 1978, Beurlen 1982) (Figs. 2, 3 e 4) e, de um modo geral, de importantes reflectores sísmicos (Fig. 5).

A seqüência carbonática basal, albo-cenomaniana, apresenta dois níveis de correlação interbacial, o inferior correspondendo ao nível de extinção do gênero Favusella na passagem Albiano Superior/Vraconiano, e o superior ao de Ticinella sp. sp. pouco abaixo do topo do Albiano (Vraconiano). Este último nível normalmente não corresponde ao topo da seqüência, cuja posição estratigráfica é algo incerta, onde, via de regra, não ocorrem associações planctônicas diagnósticas, à exceção da Bacia do Ceará, onde a presença de Praeglobotruncana delrioensis indica idade eocenomaniana (Viviers 1982 e 1985). Nos poucos poços da plataforma do Maranhão onde esta seção foi alcançada, ela se mostrou praticamente estéril em microfósseis, ocorrendo raros níveis com formas do Albiano Inferior/Médio (Hedbergella aff. H. gorbachikae e formas afins) Beurlen \& Regali, no prelo).

A porção cretácea da sequêencia "transgressiva" (Fig. 2) é caracterizada ao longo de toda a plataforma continental por três data, correspondendo aos níveis de extinção de Dicarinella concavata s.l., na passagem do Santoniano ao Campaniano, de Globotruncana fornicata s.l. na parte inferior do Maastrichtiano e de Globotruncana gansseri um pouco abaixo do topo do Cretáceo. Outro datum importante situa-se na passagem do Turoniano ao Coniaciano, representado pelos níveis de extinção de Globotruncana (?) inornata e $G$. (?) wilsoni nas bacias da margem

* Centro de Pesquisas da Petrobrás (Cenpes), Setor de Bioestratigrafia e Paleoecologia. Cidade Universitária, Quadra 7, Prédio 20, Ilha do Fundão, CEP 21910, Rio de Janeiro, RJ, Brasil 

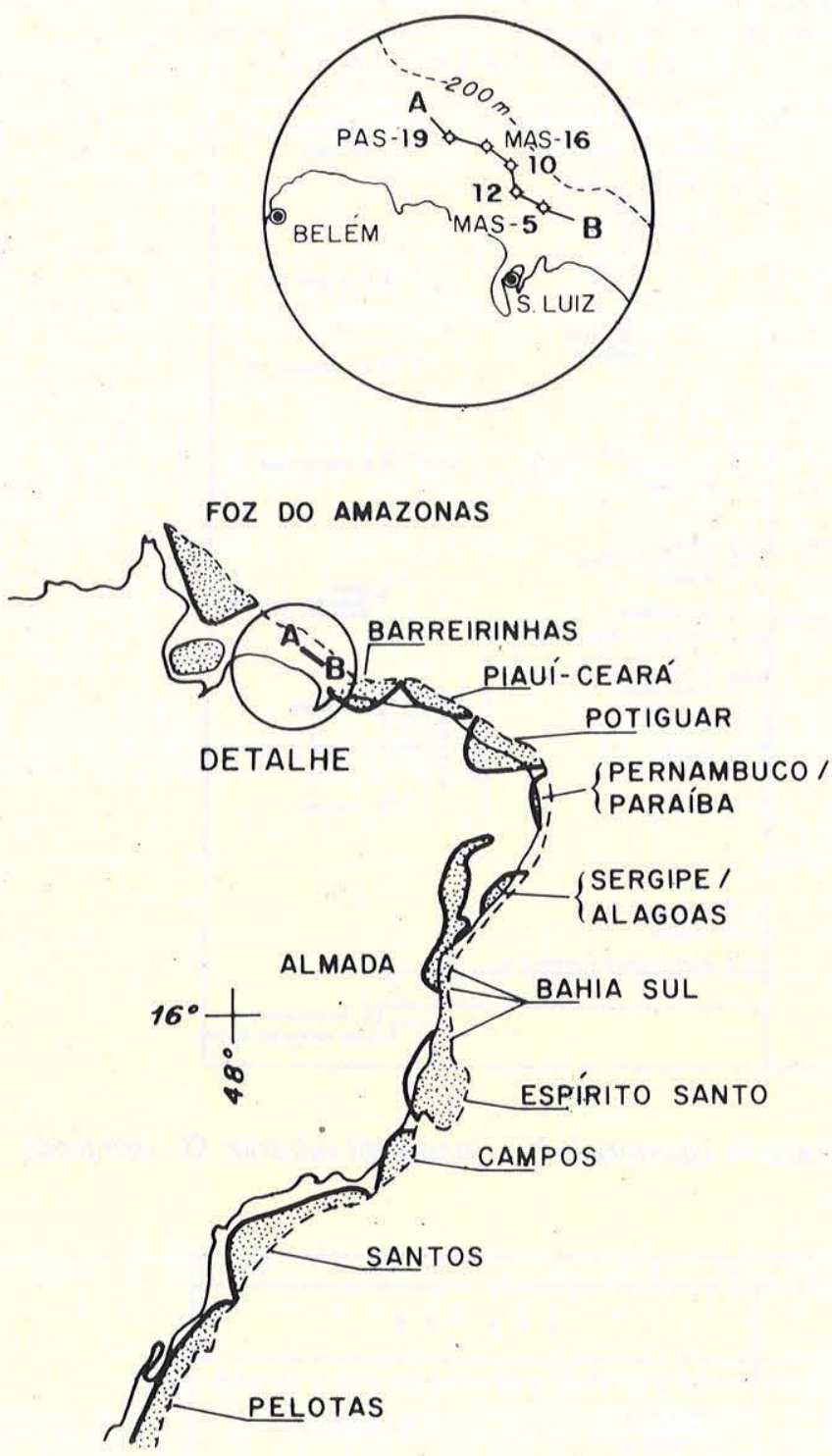

Figura 1 - Mapa de localização das bacias Mesozóico-Terciárias da margem continental brasileira

\begin{tabular}{|c|c|c|c|c|c|}
\hline \multicolumn{2}{|r|}{ IDADE } & \multicolumn{2}{|r|}{ SEQÜÊNCIA } & LITOL. & AMBIENTE \\
\hline \multicolumn{2}{|c|}{ QUATERNÁRIO } & \multirow{6}{*}{$\begin{array}{l}M \\
A \\
R\end{array}$} & \multirow{3}{*}{$\begin{array}{c}\text { PROGRADANTE } \\
\text { OU } \\
\text { REGRESSIVA }\end{array}$} & & \multirow{3}{*}{$\begin{array}{l}\text { TRANSICIONAL } \\
\text { PLATAFORMA }\end{array}$} \\
\hline \multirow{3}{*}{$\begin{array}{l}T \\
E \\
R \\
C \\
C \\
A^{\prime} \\
R \\
1 \\
0\end{array}$} & PLIOCENO & & & & \\
\hline & EOC. MÉD. & & & & \\
\hline & $\begin{array}{l}\text { EOC, INF. } \\
\text { PALEOC. }\end{array}$ & & \multirow[b]{2}{*}{ TRANSGRESSIVA } & & \multirow[b]{2}{*}{ TALUDE } \\
\hline $\begin{array}{l}C \\
R \\
\text { E } \\
\text { T }\end{array}$ & $\begin{array}{c}\text { MAASTRIC. } \\
\text { I' } \\
\text { CENOMAN. }\end{array}$ & & & & \\
\hline c & ALBIANO & & CARBONÁTICA & 독ㄱㄹ & PLATAF. \\
\hline $\begin{array}{l}\mathrm{E} \\
0 \\
\end{array}$ & $\begin{array}{l}\overline{A L} \bar{A} \bar{A} G \overline{A S} \\
\text { D. JOAOO }\end{array}$ & \multicolumn{2}{|r|}{$\begin{array}{l}\text { EVAPORITICA } \\
\text { NAOO MARINHA }\end{array}$} & & $\begin{array}{l}\text { GOLFO ' } \\
\text { FLUVIO-LACUST }\end{array}$ \\
\hline
\end{tabular}

Figura 2 - Coluna estratigráfica simplificada da seção marinha da margem continental (Beurlen 1982, modificado de Ponte et al. 1978) equatorial e de Dicarinella hagni e D. imbricata nas bacias de Campos e Santos. A parte basal do Turoniano pode ser caracterizada pela presença de uma associação de espécies de Hedbergella e Whiteinella de grande porte e ornamentação grosseira, de amplitude estratigráfica ampla, as quais, devido a condições possivelmente anóxicas com taxas relativamente altas de matéria orgânica dominam amplamente a associação planctônica (Gibbons et al. 1983, Viviers 1987).

NIVEIS DE CORRELAÇÃO BIOESTRATIGRÁFICA REGIONAL Além dos seis níveis de correlação bioestratigráfica observados ao longo de toda a margem continental, ocorre ainda uma série de outros de extensão geográfica mais restrita, úteis na correlação intrabacial ou entre bacias vizinhas. Os mais importantes destes serão brevemente discutidos a seguir.

Nas bacias de Santos e Campos, associados ao datum de Ticinella (normalmente representado por $T$. raynaudi), ocorrem Rotalipora ticinensis e $R$. appenninica, respectivamente. Na Bacia de Santos ainda são importantes: o aparecimento dos primeiros foraminíferos planctônicos bicarenados na parte superior do Cenomaniano, seguidos da ocorrência de Praeglobotruncana helvetica na parte inferior/ média do Turoniano. O ostracode Brachycythere sapucariensis também é comum em intervalos desta idade, quando desenvolvidos em ambiente relativamente raso. Nas bacias da margem equatorial e de Sergipe, a extinção desta espécie marca a passagem Santoniano/Campaniano (Sampaio \& Schaller 1968, Troelsen apud Schaller 1969).

A extinção de Globigerinelloides bentonensis, um pouco abaixo do datum de Hedbergella e Whiteinella de grande porte, marca, nas bacias do Ceará e Potiguar, a passagem Cenomaniano/Turoniano. Nestas, a extinção de Hedbergella hoelzli, muito embora de menor importância, marca o topo do Coniaciano. Na plataforma do Maranhão, a parte inferior do Santoniano é, freqüentemente, assinalada pela ocorrência de níveis ricos em Hedbergella planispira e outras formas hedbergelliformes pequenas.

O Campaniano normalmente não apresenta espécies de foraminíferos planctônicos diagnósticos, sendo melhor caracterizado por palinomorfos e nanofósseis calcários (Figs. 3 e 4).

Alguns horizontes com evidências de anoxia (Albiano Superior, Cenomaniano Superior, Turoniano e, ocasionalmente, Coniaciano e Santoniano) podem ser utilizados pelo menos em correlações intra-baciais. Normalmente, estes níveis se caracterizam pela proliferação de microfauna planctônica, ausência ou redução de associações bentônicas e taxas relativamente altas de matéria orgânica (Jenkins 1980, Dias-Brito 1987, Viviers 1987, Viviers et al. 1986).

A PASSAGEM CRETÁCEO-TERCIÁRIO Acima do datum de Globotruncana gansseri é comum ocorrer uma seção com espécies de Rugoglobigerina e Heterohelix de grande amplitude estratigráfica, cuja extinção marca comumente o topo do Cretáceo. Ocasionalmente, aparecem associadas espécies de amplitude restrita ao intervalo: Abathomphalus mayaroensis, até agora conhecida somente da bacia terrestre de Almada e de poços das bacias de Campos e Bahia Sul, e Rugoglobigerina reicheli e $R$. scotti, de distribuição geográfica mais ampla.

Nem sempre, entretanto, a passagem Cretáceo-Terciário, freqüentemente desenvolvida em ambiente de águas profun- 


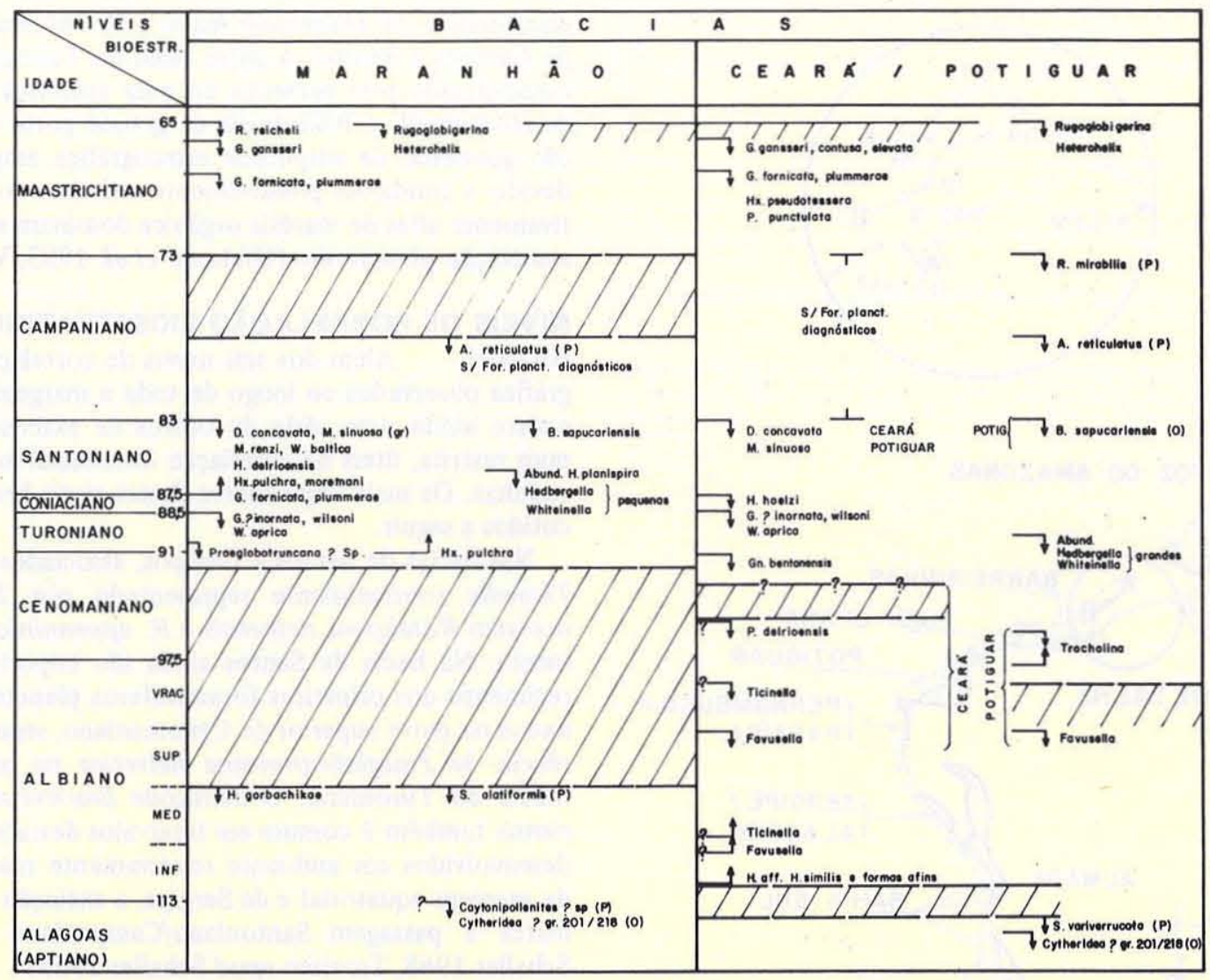

Figura 3 - Niveis de correlação bioestratigráfica de bacias da margem equatorial: $\mathbf{N}$ - nanofóssil calcário; $\mathbf{0}$ - ostracode; P - palinomorfo. (Escala de tempo segundo Harland et al. 1982)

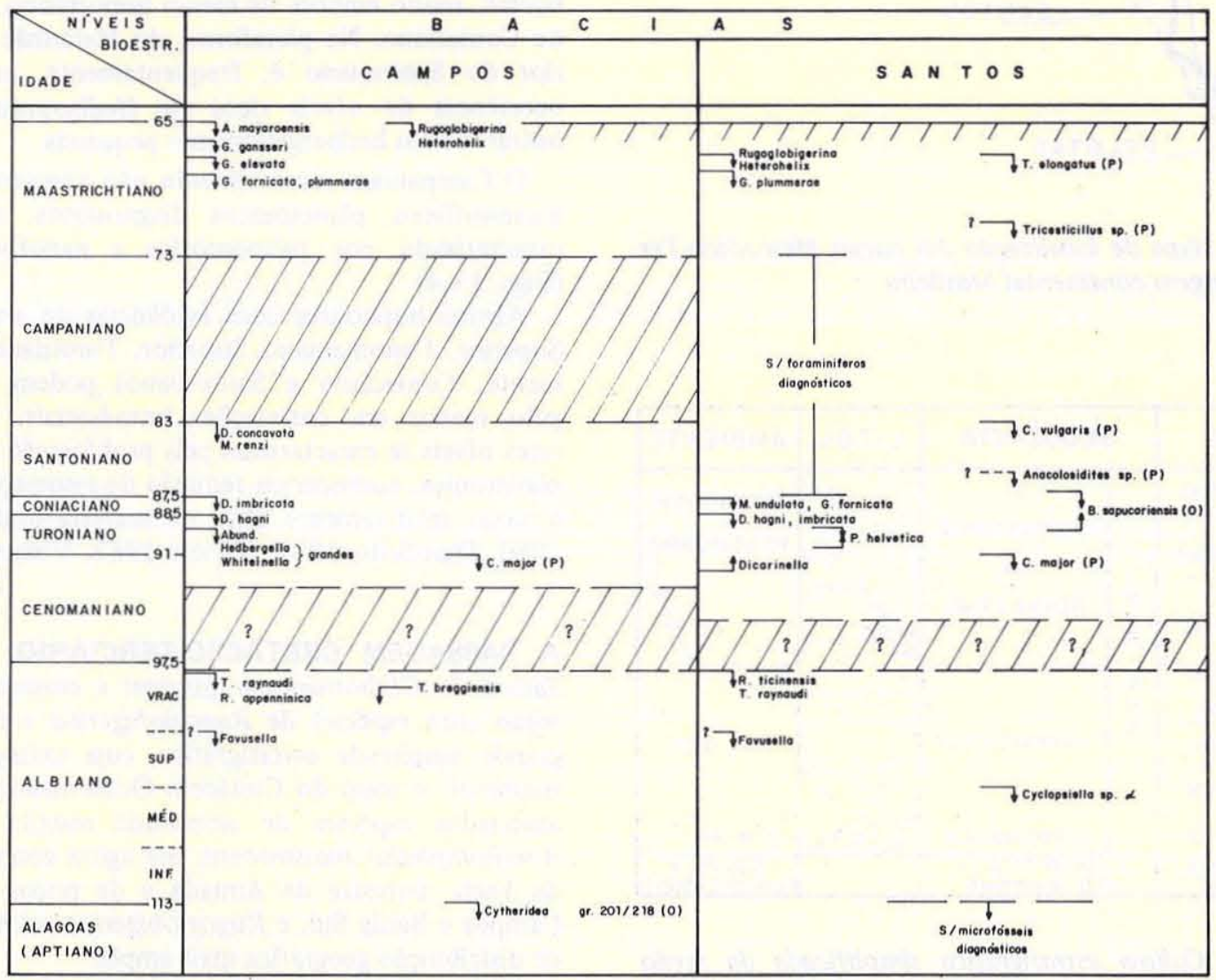

Figura 4 - Niveis de correlação bioestratigráfica de bacias da margem Leste; $\mathbf{N}$ - nanofóssil calcário; $\mathbf{0}$ - ostracode; $\mathbf{P}$ - palinomorfo. (Escala de tempo segundo Harland et al. 1982) 
das, é contínua. Falta, na maioria das vezes, a Zona Globotruncana aegyptiaca (Viviers 1982), de amplitude equivalente à porção superior da Zona Globotruncana contusa de Noguti \& Dos Santos (1978) e a de Rugoblobigerina reicheli e $R$. scotti. E comum também a ausência de sedimentos da parte basal do Paleoceno, correspondente à Zona Globorotalia (Turborotalia) longiapertura e Subzona Globorotalia (Turborotalia) pseudobulloides/Globorotalia (T.) archaeocompressa (zonas P- $\alpha$ e P-1a de Blow 1979), isto é, o intervalo caracterizado por Globigerinas primitivas (Globigerina eugubina, G. fringa).

OS HIATOS A seqüência sedimentar marinha cretácea é, habitualmente, interrompida por três hiatos reconhecidos em praticamente todas as bacias da margem continental brasileira.

O inferior, separando a seqüência carbonática albiana da seqüência "transgressiva" cretáceo-terciária (Beurlen 1982, Viviers 1982), corresponde, de modo geral, ao Cenomaniano. Aparentemente, é ligado a profundas modificações no regime sedimentar e oceanográfico do oceano Atlântico Sul e Equatorial (Melguen et al. 1978, Johnson 1983, Dias-Brito 1987). Estas mudanças são documentadas pela passagem abrupta de sedimentação de carbonatos de plataforma na seqüência da lapa para sedimentos clásticos, de águas relativamente profundas, com freqüentes aportes turbidíticos na seqüência superior (Beurlen 1982, figura 5). Isto é reflexo, evidentemente, da instalação de condições oceânicas a partir da base da seqüência "transgressiva". 0 intervalo de tempo envolvido nesta discordância varia nas diversas bacias, como conseqüência mais das características tectono-sedimentares peculiares de cada uma do que de uma queda do nível eustático do mar (Vail et al. 1977, Beurlen op. cit.).

O segundo hiato de expressão abrange o tempo correspondente ao Campaniano, mais comumente à sua parte superior. As informações disponíveis indicam a existência de um truncamento erosional na parte superior dos sedimentos de idade Santoniano/Eocampaniano, bem evidente nas bacias do Maranhão ę Campos, sugerindo que esta inconformidade possa ser relacionada com uma variação relativa do nível do mar (Fig. 5) (Beurlen \& Regali, no prelo).

A seção cretácea da seqüência "transgressiva" é limitada em seu topo por outra discordância que abrange o intervalo de tempo correspondente à porção terminal do Maastrichtiano e basal do Paleoceno.

Além destes hiatos de ampla abrangência geográfica, outros menores se registram nas diversas bacias da margem continental. Os mais bem documentados correspondem a porções do Turoniano na Bacia de Campos (Azevedo et al. 1987) e do Coniaciano do Maranhão (Fig. 5).

A discordância na base da seção marinha (Alagoas/ Albiano), apesar de sua extensão ampla, é produto de interação de múltiplos fatores geológicos, conseqüencia da instalação de condições marinhas na margem continental (Asmus 1981, Ojeda 1982). Sua análise foge ao escopo deste trabalho.

CONCLUSÕES A integração de dados bioestratigráficos de bacias da margem continental brasileira permitiu o

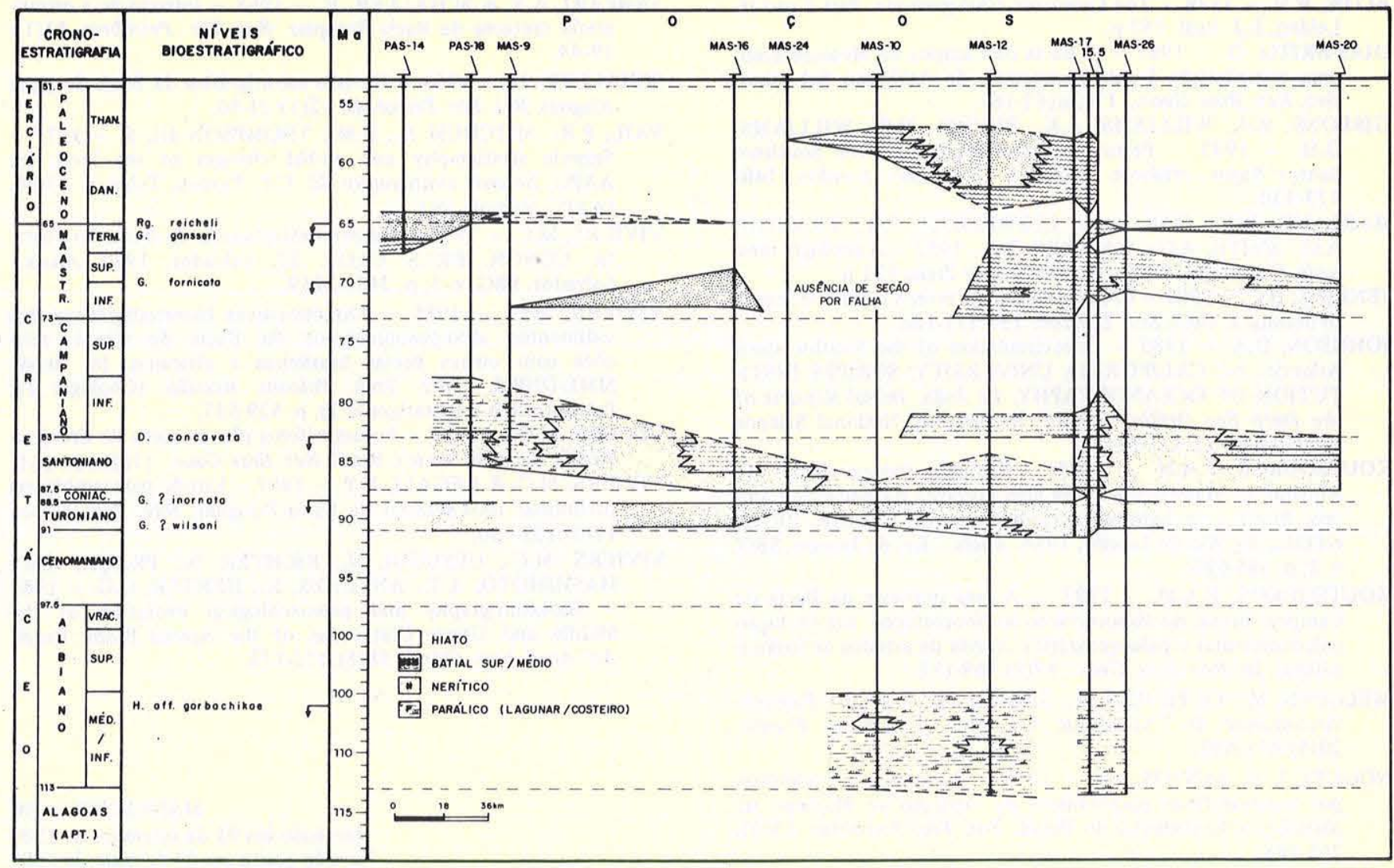

Figura 5 - Diagrama Tempo (Ma), distância longitudinal na plataforma do Maranhão. Os topos das unidades do Cretáceo correspondem a refletores sismicos datados bioestratigraficamente. (Escala de tempo segundo Harland et al. 1982) 
reconhecimento de seis data de foraminíferos planctônicos no intervalo Albiano-Maastrichtiano: nível de extinção do gênero Favusella (passagem Albiano Superior/Vraconiano) e do gênero Ticinella (pouco abaixo do topo do Vraconiano) na parte inferior da seção marinha (seqüência carbonática); e os níveis de extinção de Globotruncana ? inornata e G. wilsoni (bacias da margem equatorial) e Dicarinella hagni e D. imbricata (margem leste) no topo do Turoniano, de $D$. concavata na passagem Santoniano/Campaniano, de Globotruncana fornicata na parte inferior do Maastrichtiano e de Globotruncana gansseri pouco abaixo do topo do Cretáceo, que caracterizam a parte cretácea da seqüência "transgressiva". Permitem boa correlação tanto interbacial como mundial.

Quatro discordâncias foram reconhecidas nas bacias da margem equatorial, Campos e Santos: na base da seqüência carbonática (Alagoas/Albiano), entre esta e a seqüência "transgressiva" (Cenomaniano) e mais duas dentro desta (Campaniano e passagem Cretáceo-Terciário). Enquanto as duas primeiras parecem ser controladas principalmente pela interação de fatores tectono-sedimentares da fase pós-rift da abertura do Atlântico Sul e Equatorial, a ocorrência de truncamento erosional na parte superior dos sedimentos santoniano-campanianos e do Maastrichtiano Superior sugere que, nas duas últimas, variações do nível eustático do mar tenham exercido papel importante.

Agradecimentos Os autores agradecem à Diretoria da Petróleo Brasileiro S.A., pela permissão de publicação, e ao geólogo Jorge Carlos Della Fávera, pelas estimulantes discussões e sugestões durante a leitura do manuscrito.

\section{REFERÊNCIAS BIBLIOGRAFICAS}

ASMUS, H.E. - 1981 - Geologia das bacias marginais atlânticas mesozóicas-cenozóicas do Brasil. In: COMITE SUDAMERICANO DEL JURASICO \& CRETACICO DE AMERICA DEL SUR. Cuencas Sedimentarias del Jurasico y Cretacico de America del Sur. Buenos Aires, p. 127-155.

AZEVEDO, R.L.; GOMIDE, J.; VIVIERS, M.C.; HASHIMOTO, A.T. - 1987 - Bioestratigrafia do Cretáceo marinho da Bacia de Campos. Rev. Bras. Geoc., 17(2):139-146.

BEURLEN, G. - 1982 - Bioestratigrafia e geohistória da seção marinha da margem continental brasileira. Bol. Téc. Petrobrás, 25(2):77-83.

BEURLEN, G. \& REGALI, M.P. O Cretáceo da plataforma do Maranhão; bioestratigrafia e evolução paleoambiental. Bol. Téc. Petrobrás. (no prelo).

BLOW, W.H. - 1979 - The Cainozoic Globigerinida. Part I and II. Leiden, E.J. Brill. 752 p.

DIAS-BRITO, D. - 1987 - A Bacia de Campos no Mesocretáceo: uma contribuição à paleoceanografia do Atlântico Sul primitivo. Rev. Bras. Geoc., 17(2):162-167.

GIBBONS, M.J.; WILLIAMS, A.K.; PIGGOT, N.C.; WILLIAMS, G.M. - 1983 - Petroleum geochemistry of the Southern Santos Bașin, offshore Brazil. J. Geol. Soc. London, 140: 423-430.

HARLAND, W.B.; COX, A.V.; LLEWELLYN, P.G.; PICKTON, A.G. SMITH, A.G.; WALTERS, R. - $1982-$ A geologic time scale. Cambridge, Cambridge University Press, $131 \mathrm{p}$.

JENKINS, H.C. - 1980 - Cretaceous anoxic events from continents to oceans. J. Geol. Soc. London, 137:171-188.

JOHNSON, D.A. - 1983 - Paleocirculation of the Southwestern Atlantic. In: CALIFORNIA UNIVERSITY, SCRIPPS INSTITUTION OF OCEANOGRAPHY, La Jolla. Initial Reports of the Deep Sea Drilling Project. Washington, National Science Foundation, 72:977-994.

KOUTSOUKOS, E.A.M. - 1984 - Evolução paleoecológica do Albiano ao Maastrichtiano na área noroeste da Bacia de Campos, Brasil, com base em foraminíferos. In: CONGR. BRAS. GEOL., 33, Rio de Janeiro, 1984. Anais... Rio de Janeiro, SBG. v. 2, p. 685-698.

KOUTSOUKOS, E.A.M. - 1987 - A área noroeste da Bacia de Campos, Brasil, do Mesocretáceo ao Neocretáceo: sua evolução paleoambiental e paleogeográfica através de estudos de foraminíferos. In: Rev. Bras. Geoc., 17(2):168-172.

MELGUEN, M.; LE PICHON, X.; SIBUET, J.C. - 1978 - Paléoenvironnement de l'Atlantique sud. Bull. Soc. Geol. France, 20(4):471-489.

NOGUTI, I. \& SANTOS, J.F. - 1978 - Zoneamento preliminar por foraminíferos planctônicos do Aptiano ${ }^{\circ}$ ao Mioceno na plataforma continental do Brasil. Bol. Téc. Petrobrás, 15(3): 265-283.
OJEDA, H.A.O. - 1982 - Structural framework, stratigraphy and evolution of brazilian marginal basins. AAPG Bull., 66(6):732-749.

PONTE, F.C.; DAUZACKER, M.V.; PORTO, R. - 1978 - Origem e acumulação de petróleo nas bacias sedimentares brasileiras. In: CONGR. BRAS. GEOL., 30., Recife, 1978. Anais... Recife, SBG. v. 2, p. 121-147.

REGALI, M.P. - 1980 - Palinoestratigrafia da Bacia do Ceará. In: CONGR. BRAS. GEOL. 31, Baln. Camboriú, 1980. Anais... Baln. Camboriú, SBG.v. 5, p. 3118-3129.

RICHTER, A.J. - 198.7 - Subafloramento das discordâncias turoniana e campaniana no sul da Bacia de Campos, Brasil. Rev. Bras. Geoc., 17(2):173-176.

SAMPAIO, A.V. \& SCHALLER, H. - 1968 - Introdução à estratigrafia cretácea da Bacia Potiguar. Bol. Téc. Petrobrás, 11(1): 19-44.

SCHALLER, H. - 1969 - Revisão estratigráfica da Bacia Sergipe/ Alagoas. Bol. Téc. Petrobrás, 12(1):21-86.

VAIL, P.R.; MITCHUM Jr., R.M.; THOMPSON III, S. - 1977 Seismic stratigraphy and global changes of sea level. In: AAPG. Seismic stratigraphy Ed. C.E. Payton. Tulsa, p. 83-98. (AAPG Memoir, 26).

VIVIERS, M.C. - 1982 - Biocronoestratigrafia da Bacia do Ceará. In: CONGR. BRAS. GEOL. 32, Salvador, 1982. Anais... Salvador, SBG. v. 5, p. 2433-2449.

VIVIERS, M.C. - 1985 - Características bioestratigráficas dos sedimentos albo-cenomanianos da Bacia do Ceará: relações com outras bacias brasileiras e africanas. In: Brasil. MME-DNPM. Colet. Trab. Paleont. Brasília. (Geologia 27, Paleontologia e Estratigrafia 2), p. 529-537.

VIVIERS, M.C. $\rightarrow 1987$ - Foraminíferos planctônicos do Cretáceo Médio, Bacia de Santos, Brasil.Rev. Bras. Geoc., 17(2):154-161.

VIVIERS, M.C. \& REGALI, M.P. - 1987 - Estudo paleoambiental preliminar no Cretáceo da Bacia Potiguar. Rev. Bras. Geoc., 17(2):123-130.

VIVIERS, M.C.; UESUGUI, N.; RICHTER, A.; PRAÇA, U.M.; HASHIMOTO, A.T.; ANTUNES, R.; HERTER, G.G. - 1986 - Bioestratigraphy and paleoecological evolution of the Middle and Upper Cretaceous of the Santos Basin. Brasil. An. Acad. bras. Ciênc., 58(1):172-173.
MANUSCRITO 390

Recebido em 01 de setembro de 1986 Revisão aceita em 29 de maio de 1987 